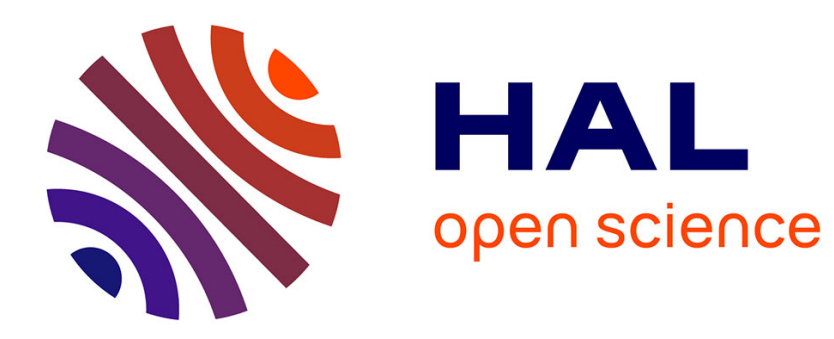

\title{
LA CRÉATION AU RISQUE D'INTERNET
}

\author{
Jean-Paul Fourmentraux
}

\section{To cite this version:}

Jean-Paul Fourmentraux. LA CRÉATION AU RISQUE D'INTERNET: MOUCHETTE (19962006): (EUVRE ET/OU ARTISTE?. Ethnologie française, 2008, 2008/1 (38), pp.61-71. 10.3917/ethn.081.0059 . halshs-00372585

\section{HAL Id: halshs-00372585 https://shs.hal.science/halshs-00372585}

Submitted on 1 Apr 2009

HAL is a multi-disciplinary open access archive for the deposit and dissemination of scientific research documents, whether they are published or not. The documents may come from teaching and research institutions in France or abroad, or from public or private research centers.
L'archive ouverte pluridisciplinaire HAL, est destinée au dépôt et à la diffusion de documents scientifiques de niveau recherche, publiés ou non, émanant des établissements d'enseignement et de recherche français ou étrangers, des laboratoires publics ou privés. 


\section{LA CRÉATION AU RISQUE D'INTERNET}

MOUCHETTE (1996-2006) : CEUVRE ET/OU ARTISTE?

Jean-Paul FOURMENTRAUX

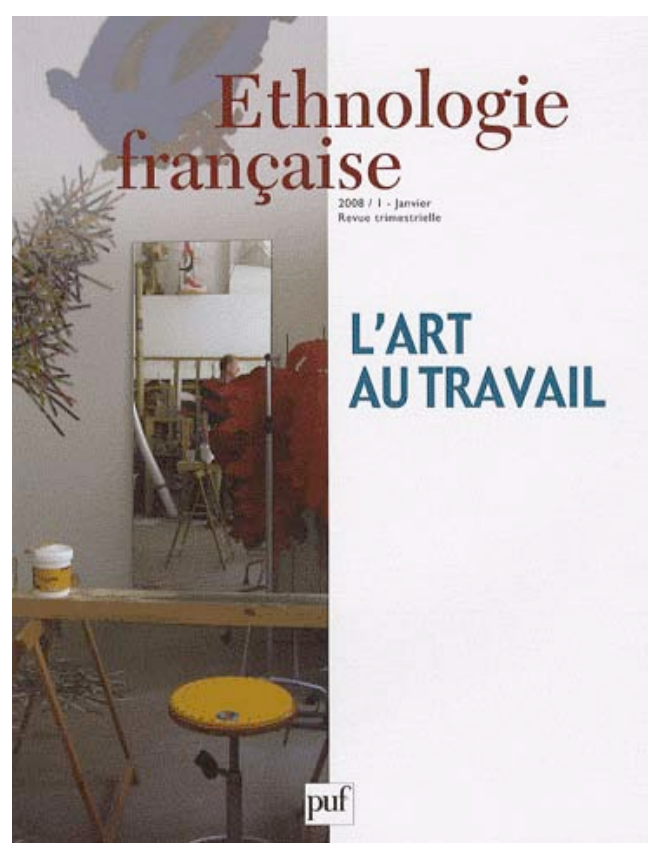

Version "auteur" avant parution. Pour toute citation, toujours se référer à l'article : FOURMENTRAUX JP. 2008, "La création artistique au risque d'Internet. Mouchette (1996-2006) : œuvre et/ou artiste ? ", Ethnologie Française, XXXVIII, 2008/1, spécial "l'art au travail" pp. 61-71.

Article consultable et téléchargeable sur le portail CAIRN: http://www.cairn.info/revue-ethnologie-francaise-2008-1.htm 


\title{
Résumé
}

Internet transforme les processus de définition d'une activité ou d'une œuvre comme « artistique » et les manières dont les créateurs y vivent, y façonnent et y affirment leur identité d'artiste. Cet article propose l'ethnographie d'un projet développé à l'interface d'Internet et des mondes de l'art contemporain : Mouchette (1996-2006). Tout en mettant l'accent sur l'ambivalence de son inscription clivée entre le réseau et la scène artistique, il s'agit d'en exposer les paramètres interactifs de production, qui oscillent entre les initiatives de l'artiste, les innovations informatiques et les interactions avec le public.

Mots-clés : travail artistique et identité, internet, mondes de l'art, Euvre, auteur.

\begin{abstract}
Internet transforms the definition of an activity or work qualified as "artistic". It also transforms the way in which creators live, the way they build and assert their identity as artists. This paper proposes the ethnography of a project carried out at the interface of Internet and contemporary artworlds : Mouchette (1996-2006). Focusing on the question of the ambivalence of his divided, dual inscription, between the network and the artistic scene, the paper sheds light on the interactive parameters of production, between the initiatives of the artist, computer innovations and interactions with the public.
\end{abstract}

Keywords : artistic work and identity, internet, artworlds, artworks, author. 
Depuis une dizaine d'années, le Net art s'impose en France où il désigne les créations interactives conçues par, pour et avec le réseau Internet, en les distinguant des formes d'art plus traditionnelles simplement transférées sur des sites-galeries et autres musées virtuels. Pour les mondes de l'art, l'originalité d'Internet tient à ce qu'il propose simultanément un support, un outil et un environnement créatif. Il faut entendre par support sa dimension de vecteur de transmission, dans le sens où Internet est son propre diffuseur ; par outil, sa fonction d'instrument de production, qui donne lieu à des usages et génère de nouveaux produits artistiques ; et par environnement, enfin, le fait qu'il constitue un espace habitable et habité. Dans ce contexte, le travail artistique vise la conception de dispositifs interactifs, mais aussi la production de formes de communication et d'exposition visant à impliquer l'internaute dans le procès de l'œuvre [Fourmentraux, 2005]. Utilisant toutes les fonctionnalités d'Internet - le Web (l'html, le ftp, le peer to peer) mais aussi le courriel, le chat - le Net art promeut des œuvres dont les enjeux relationnels et collaboratifs bousculent les relations entre art et société. Le site Internet, la homepage, le blog, les mailing lists ou les forums de discussion constituent en effet les cadres de sociabilités renouvelées. Le réseau Internet est tout autant investi comme un atelier en ligne que comme un lieu d'exposition : c'est-à-dire comme l'espace de création, de communication et de mise en œuvre de la pratique artistique. Les œuvres qui en résultent sont par conséquent des projets multiformes - environnements navigables, programmes exécutables, formes altérables - qui vont parfois jusqu'à inclure une possibilité d'apport ou de transformation du matériau artistique initial.

Autrement dit, le milieu que constitue Internet transforme conjointement les processus de définition d'une activité ou d'une œuvre comme « artistique » et les manières dont les créateurs y vivent, y façonnent et y affirment leur identité d'artiste. Afin d'en rendre compte, cet article propose l'ethnographie d'un projet développé à l'interface d'Internet et des mondes de l'art contemporain : Mouchette (1996-2006). En mettant précisément l'accent sur 
l'ambivalence de son inscription clivée entre le réseau et la scène artistique, il s'agit d'en exposer les paramètres interactifs de production, entre initiatives de l'artiste, innovations informatiques et interactions avec le public. L'analyse repose sur le suivi ethnographique de cette " mise en œuvre d'art » et rend compte des « rapports au public » que la prise en charge artistique du médium Internet peut introduire, engager et promouvoir. Ce texte s'inscrit ainsi à la suite de recherches qui se sont donné pour objet d'étude l'articulation des faits techniques et sociaux, non sur le mode de l'instrumentation ou de l'aliénation, mais sur celui de la fréquentation et du contact, voire du jeu [Akrich, 1990, 1993 ; Dodier, 1994 ; Duguet, 2002]. En s'appuyant sur une série d'entretiens et d'échanges par courriel avec Mouchette, l'article interroge également l'impact de ces mutations du travail artistique sur le statut d'artiste et pour la définition de l'œuvre entre le réseau Internet et les mondes de l'art. L'enjeu est aussi méthodologique, à travers la mise en perspective de manières de procéder à une ethnographie du travail artistique [Buscatto, 2006] centrée sur la « carrière » d'un projet de création et attentive aux processus pragmatiques et/ou sociotechniques de sa « mise en œuvre d'art ». Dans ce contexte, une ethnographie du travail artistique n'implique nullement de s'intéresser à l'œuvre exclusivement comme un objet déjà constitué. Replacée au cœur du drame social du travail artistique [Hughes, 1996], l'œuvre d'art y est sociologiquement analysée comme le nœud d'activités collectives et de négociations mises en jeu à l'interface de différents mondes sociaux dans lesquels elle fait sens et se déploie [Becker, 1988 ; Dodier, 1991 ; Strauss, 1992]. L'enjeu est également réflexif : à partir de l'observation empirique des mécanismes et dispositifs de mise en œuvre d'art [Duguet, 1988 ; Fourmentraux, 2005] et de construction de l'identité d'artiste, il s'agit de tirer des enseignements utiles aux connaissances des récentes mutations du travail de création par et dans l'environnement Internet. 


\section{- La rencontre avec Mouchette (1996-2001) : encadré méthodologique}

L'observation ethnographique des activités de «mise en œuvre d'art » dans le contexte de l'Internet demande de saisir de front les configurations techniques, les constructions identitaires, les formes d'attachement du public, l'ensemble des rituels sociotechniques qui composent l'originalité et la spécificité de ces créations interactives. Dans le cas de Mouchette, cette analyse repose sur une fréquentation assidue du site Web, ponctuée de nombreux et réguliers échanges par courriels, d'un recueil et d'une analyse systématique des articles, chroniques journalistiques et nombreux débats qui ont accompagné la «publicisation » de Mouchette. En faisant varier les modes d'implication, de l'internaute fidélisé à l'ethnographe déclaré, l'enquête déploie un large éventail de matériaux et de techniques audiovisuelles qui mettent au jour les conditions et modalités pratiques de la relation interactive - aux interfaces, dispositifs, images et textes, courriels adressés, etc. - qui organisent les parcours et interactions avec Mouchette. Mais l'enquête s'appuie également sur une série d'entretiens, réalisés d'abord par courriel, sous la forme d'une conversation et d'un échange régulier de questions/réponses, sur plusieurs années, suivi d'un entretien en face à face, réalisé lors du festival international de création vidéo de Montbéliard, à Belfort en 2001 (Festival Interférences). C'était alors la première fois que l'artiste acceptait de (re)présenter Mouchette devant une audience " restreinte ", réservée aux animateurs et aux artistes du festival. L'ensemble de ce matériau empirique est issu d'une enquête effectuée dans le cadre plus général d'une thèse de sociologie [Fourmentraux, 2003, 2005].

\section{Cuvre/identité virtuelle et art de la captation}

Dans l'univers des médias, un engagement plus « expressiviste » se lit aujourd'hui à travers l'essor et la multiplication des dispositifs d'autoproduction ou de production de soi que constituent les sites personnels, les blogs et leurs technologies appareillées (syndication et tags, 
podcasting, video-blogging...) ainsi que les réseaux d'échanges entre pairs et leurs pratiques associées (fansubbing, fansfilms...). Depuis la seconde moitié des années 1990, le Net art guide et préfigure ces usages de l'Internet grand public à travers une mise en abîme de l'ambivalence de la relation au réseau, tantôt intime et terriblement solitaire, vécue comme un retrait du monde réel, tantôt plus collective et communautaire, à mesure que se développent de nouvelles interfaces de dialogue. Exemplaire de ce mouvement, Mouchette [1] se présente sur le Web comme un avatar inspiré du film éponyme de Robert Bresson (1967 [2]), auto-(re)produisant ainsi depuis 1996 les aléas et la personnalité d'une adolescente hollandaise confrontée aux périls du suicide et de la pornographie pédophile. Le site transpose sur Internet le "genre » de la page personnelle en tant que construction fantasmatique, suivant la logique d'un journal intime qui met en scène, sur un ton de provocation, les frontières entre vie artistique et vie privée [3].

Au-delà de son esthétique volontairement kitsch, contrastée par une succession d'images et de sons toujours plus obscurs et troublants, une première spécificité et un ressort important de Mouchette résident dans l'établissement d'un échange personnalisé, visant à construire et à entretenir, à l'écart de toute médiation extérieure, une liaison quasi amoureuse avec le public. À cet égard, l'expérience de Mouchette permet à l'internaute, et au sociologue, d'entrevoir et de comprendre comment le Net art parvient à créer une relation de proximité et un rapport intime entre un dispositif interactif et celui qui le consulte. La problématisation des liens entre fiction et réalité, l'invention d'un personnage virtuel et la question de l'identité artistique constituent les thèmes de prédilection de Mouchette dont l'enjeu est de redéployer sur le réseau la base du concept de personnage, ses fonctions, ses programmes, ses modes de relation avec le public. 


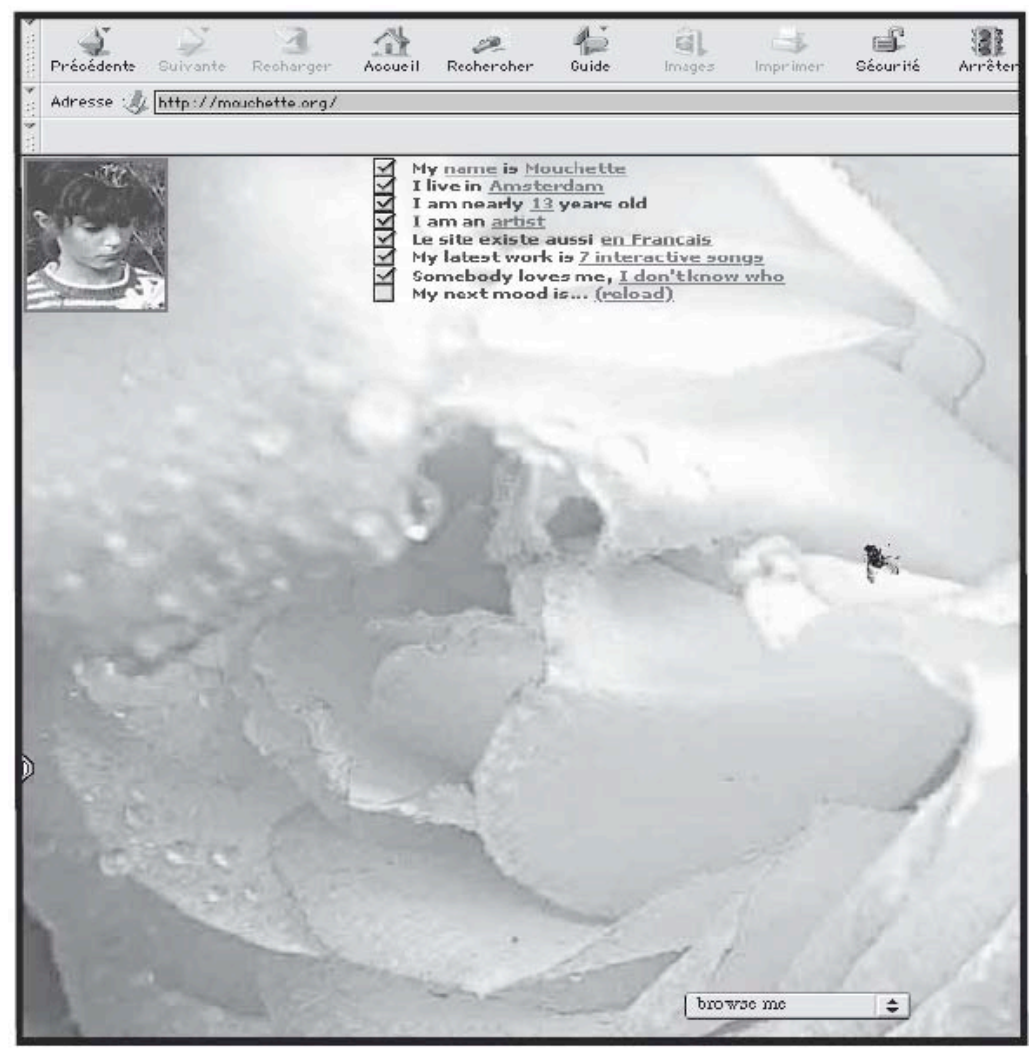

Mouchette, Page d'accueil, http://www.mouchette.org

«C'est un outil de communication, il permet, il transforme ou il détourne les échanges entre les usagers d'Internet. Mon personnage n'est pas là pour être contemplé, il se situe en plein cœur du flot des informations et modifie cette circulation. Il est à la disposition du public qui s'en sert et le fait vivre. » (Entretien avec Mouchette, 2001.)

La stratégie artistique mise en œuvre consiste à jouer du caractère performatif du langage afin de susciter l'implication et les actes du public. Une performativité de l'acte de langage au sens de John Austin (1970) selon lequel le sujet qui prononce effectue une action, dans le moment et par le fait même qu'il prononce. Cette stratégie est en effet fondée sur un double procédé de séduction du public et d'adressage personnalisé qui aménage, 
voire impose, une possibilité d'agir à travers l'envoi de courriels qui sont intégrés à l'œuvre et forment un cadre d'échange avec les visiteurs successifs du site Internet. L'enjeu du dispositif réside par conséquent dans l'établissement d'une relation « personnalisée » avec chacun des visiteurs.

« Il y a l'adresse directe par le texte, c'est-à-dire que qui dit "Je" est une personne. C'est une sorte d'axiome. En fait, il n'y a pas de personne. Il n'y a pas de Mouchette. Il y a quelque chose qui dit "Je" et qui s'adresse, qui dit "Tu". Donc cela crée une sorte d'illusion de personnalité qui fonctionne et qui crée un certain état de réception sur le spectateur. » (Entretien avec Mouchette, 2001.)

Habilement agencé par l'artiste, ce processus conversationnel est automatisé à travers le langage de programmation php3 : un programme informatique enregistre et mémorise les éléments d'informations fournis dans les courriels envoyés par le public. Le nom et l'adresse, la date, l'heure du message et son contenu sont analysés, répertoriés et classés selon des catégories de réponses. La technologie des formulaires en php3 rend ensuite possible, depuis le serveur Internet de l'artiste, la création d'une base de données qui récupère ces informations et crée une liste d'envoi de différentes interventions personnalisées : la part immergée du dispositif consistant en une base de données où sont archivées les contributions successives des visiteurs, leurs adresses ainsi qu'un historique de leur participation.

«Une partie est automatisée parce que bien sûr je ne peux pas écrire trois mille mails par jour. Cette manière de communiquer avec son public fait partie de l'art. Donc, la médiation n'est pas séparée de la production. La médiation est l'œuvre. Il n'y a pas de différence. » (Entretien avec Mouchette, 2003.)

La propagation de l'œuvre et la captation du public [Cochoy, 2004] apparaissent par conséquent dans ce dispositif comme deux processus conjoints qui constituent pour l'artiste une part majeure du travail créatif. Mouchette possède aujourd'hui son fan-club, véritable répertoire de 
participants fidélisés au fil des échanges réguliers par courriels et autres privilèges dont ils ont l'exclusivité [4].

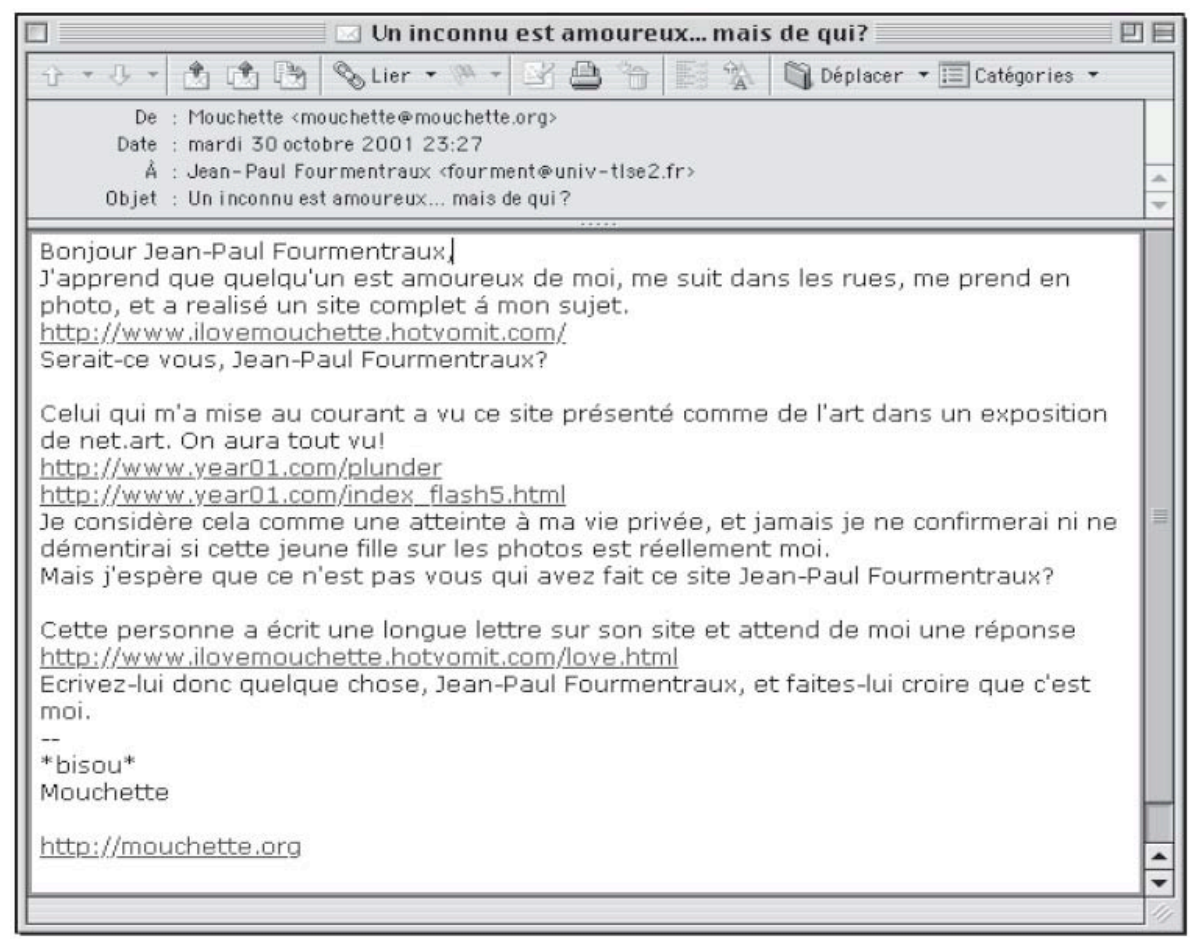

Mouchette, Dispositif de captation par adressage personnalisé

\section{Le Net art et son public : de l'accès à la participation}

Mouchette invite le public des internautes non seulement à naviguer dans une fiction dont les ramifications multiples se déploient de page en page, mais aussi et surtout à accomplir une série d'actions qui ont des influences directes sur le contenu de ces fictions. Lorsque Mouchette propose une création collective le public impliqué et participant devient luimême un personnage et se trouve ainsi inscrit dans la fiction. Mouchette manipule le public et dialogue avec lui de diverses manières, en le perdant dans un dispositif narratif alambiqué, en l'invitant à « Tuez le chat ! » d'un 
simple clic de souris, pour mieux s'en plaindre (pleurnicher) et regretter ensuite les conséquences terribles de cet acte [5] : le public s'en trouvera luimême d'abord surpris puis affecté d'un vif sentiment de culpabilité. Cela fonctionne d'autant mieux ici que les thèmes abordés par l'œuvre interrogent simultanément le vécu du visiteur et les effets de son implication face à l'ordinateur. Ce penchant promeut une sorte de Web-réalité où le public (voyeur) est parfois conduit à décider du sort du personnage ou de l'action. En attribuant aux actions des regardeurs (matérialisées par le clic de souris) des conséquences irrémédiables qui s'inscrivent dans le récit et en modifient le cours, Mouchette met en scène l'activité de "réception » qui devient partie prenante de l'œuvre. Le public y est en première instance renvoyé aux conséquences de ses actes, qu'il s'agisse de donner la mort ou l'amour au travers de sa relation à l'œuvre "médiée » par l'écran. Ses « réactions » riches et éloquentes se trouvent également, dans un second temps, mises en scène dans le dispositif artistique. À mesure qu'il découvre les ressorts de la fiction et conscient de participer à un jeu, le public redéploie à son tour le récit et justifie son acte en faisant parfois preuve d'une nouvelle créativité, jouant du deuxième ou du troisième degré. Au «pourquoi ? » plaintif de Mouchette, il répondra «parce que je suis un tueur de chats!» ou " parce que je suis un chien! ", et parfois même, par une mise en abîme de l'acte de langage et du programme informatique, «parce que ton code est mal écrit ! ». Le dispositif met ainsi simultanément en scène les actes et les réponses du public en jouant du décalage entre un graphisme et une structure ludique simplifiée et la complexité de l'algorithme informatique qui prend en charge l'organisation de ces contenus, à lectures multiples, qui font appel à différents niveaux de connaissance de celui ou celle qui interagit.

Cette étreinte et inculpation du public engage une posture de réflexivité interactive et responsabilise le public face à ses " actes », si virtuels puissent-ils être. La stratégie et la condition de ce projet créatif reposent par conséquent sur l'acceptation par le public d'une mythographie artistique et d'une identité fabriquée pour qu'il se prête au jeu et libère ses émotions en 
ajoutant ses propres fantasmes à la construction du personnage. À la croisée de l'œuvre communicationnelle et de la performance, le dispositif instaure de la sorte un rituel de lecture disséminé dans le temps et dans les arcanes des réseaux, dont l'initiation appelle des degrés variés de participation. La circulation de l'œuvre repose sur des dispositifs d'implication du public qui se trouvent (en effet) incarnés et mis en scène dans des programmes informatiques qui règlent différents modes d'interactivité, d'une part, et à travers des stratégies contractuelles [Passeron et Pedler, 1991] [6] de fidélisation du public qui aménagent diverses prises [Bessy et Chateauraynaud, 1998] [7] sur l'œuvre, d'autre part. Face à l'altérité des publics qui caractérise Internet, Mouchette témoigne ainsi de manière particulièrement aiguë de la volonté artistique - qui est en même temps une crainte - de rencontrer (ou non) sur Internet une audience élargie et renouvelée.

Le caractère innovant de sa mythographie, qui articule à une réalité fantasmée une interaction négociée avec le public, fait de Mouchette une des artistes Web les plus représentatives de l'Internet créatif. Il semble de ce point de vue que le public y adhère autant pour le «contenu » qu'il découvre sur le site Mouchette que parce que ce dernier lui accorde un rôle et un statut original dans le processus de sa mise en œuvre.

\section{Les frontières de l'œuvre : Internet et mondes de l'art}

Le caractère semi-public semi-privé d'Internet favorise ainsi des réalisations couvrant le thème de l'intimité et de la communication interactive. Mais de tels projets connaissent des fortunes diverses : certains sont plébiscités sur le réseau et restent le plus souvent inconnus des mondes de l'art ; d'autres en revanche parviennent peu ou prou à jouer de cette frontière encore très peu poreuse entre la sphère de l'Internet et la scène de l'art. Mouchette constitue un cas exemplaire de cette difficile circulation entre les deux mondes, ou d'un monde à l'autre. Son projet prend corps dans 
le réseau, où il puise ses ressources et où il côtoie (à un ou deux clics de souris) quantité d'autres sites, dont les finalités sont très éloignées. Internet apparaît ici comme un territoire ambigu, habité par des publics hétérogènes, confrontant des fragments de réalité et des activités sociales multiples. Par la création de collectifs et de situations communicationnelles, Mouchette donne forme à des rituels de consommation culturelle à l'écart des lieux et des milieux traditionnels de la diffusion artistique. Le site n'offre en effet aux internautes aucun repère ou indice a priori de son objet ou de son appartenance à la sphère des arts. Son dispositif exploite au contraire des formes de cohabitation et de contamination entre différents ordres et niveaux de réalité : de la réalité déviante des pirates de l'informatique (hackers) à celle des graphistes (Web designers) et des publics amateurs de «pages personnelles » qui arrivent sur ce site souvent par accident. Le site Web Mouchette attire quotidiennement des centaines et parfois des milliers de visiteurs. La relation au public y est maintenue de manière exclusivement digitale par l'intermédiaire du site Web et du courriel. Des mises à jour régulières du site renouvellent son actualité, ses parties interactives suscitant d'abondantes réactions et participations. L'œuvre connaît parallèlement une très forte valorisation dans les réseaux de l'art électronique et numérique déployés sur le Web à travers des plates-formes influentes et reconnues comme Rhizome ou Hell.com, qui comptent parmi les membres du fan-club de Mouchette.

Un événement survenu dans le courant de l'année 2002 rend particulièrement visibles ces nouveaux réseaux de pairs mobilisés autour de la « défense " du Net art. La Société des auteurs et compositeurs dramatiques (sacd), à la demande de Mme veuve Bresson, a le 23 juillet 2002 interdit à Mouchette de diffuser sur le Web une de ses œuvres qui fait référence au film de Robert Bresson créé en 1967 et intitulé « Mouchette ». L'œuvre interdite est un quiz qui compare les deux personnages, celui du film de Bresson et celui du site http://mouchette.org. Mais le Quiz, une fois retiré du site Mouchette, va se voir hébergé sur un nombre important de 
sites-miroirs voués au Net art, par des pairs indignés devant la procédure d'interdiction, alors qualifiée de "censure » à la liberté d'expression ou de création sur le réseau Internet [8]. En soutien à Mouchette, considérée au contraire comme un « hommage » à l'œuvre de Robert Bresson (1967), un appel fut adressé à de nombreuses listes de diffusion à destination des communautés de Net artistes. Ces derniers décidèrent de relayer l'information sur leur propre site et surtout d'héberger une copie du Quiz afin d'en assurer la perpétuation en de multiples lieux et de rendre ainsi caduque toute velléité d'interdiction [9]. À cet égard, le curriculum vitae de Mouchette publié en ligne est éloquent : quoique le concepteur du site Web reste inconnu, l'œuvre ne manque pas de faire l'objet de nombreuses représentations et diffusions à travers une imposante variété de sites Web, artistiques ou non, de festivals et d'expositions en ligne, où elle reçoit de ses pairs de nombreux prix. Au-delà de la gestion particulière de la relation au public que nous avons précédemment analysée, l'Internet favorise en effet la création de nouveaux réseaux artistiques constitués de nombreuses revues critiques, de festivals spécialisés dans les arts numériques, de forums et de listes de diffusion qui ponctuent et organisent la vie du Net art. 


\section{Artistic events}

*March 06: "DorkBotToronto - event and presentation Interaccess, Toronto, Canada

^Aug 05: "Virtuelle Identitäten" Ladyfest, Berlin, Germany

*May 05: Flash Festival "intime ${ }^{\star} 10^{"}$ Centre Pompidou Paris France

^August 04: KUNSTNETZNRW.DE, Virtuelle Galerie

`March 04: WEBeSCAPE, in Palermo, Italy; web room Mouchette

*March 04: Digitalia, curated by Michele Thursz, Wood Street Gallery, Pittburgh

*March 04: SYZYGY (the Human Remix), Art In Motion Festival, University of Southern California

*Jan Imay 04: P2P[iece] Project, POSTARTUM, Long Beach California

*Jan 04:Medi@terra festival 6, Athens, Greece

Extrait du curriculum vitae en ligne : http://mouchette.org/cv/

À l'image des galeries alternatives qui ont accompagné le développement de l'art vidéo dans les années 1970, le Net art promeut à son tour l'émergence de lieux où se réalise l'agencement des productions et leur autopromotion. Internet est alors envisagé comme un environnement au sein duquel peut se déployer, au quotidien, l'activité créative : les sites Web offrant un nouveau cadre de travail aux artistes qui partagent cet espace y invitent et y reçoivent des propositions d'autres artistes, ainsi que les contributions des internautes, leurs commentaires, etc. La métaphore de l'« atelier en ligne » constitue la principale figure de ce régime domiciliaire du Net art. En défaisant le coutumier clivage entre l'atelier et la galerie (ou le musée), Internet expose au regard du public l'espace intime où se déploient les ficelles du métier et fait émerger à l'écran le savoir-faire et les œuvres inachevées de l'artiste. D'une part, l'atelier dans sa version on-line se propose comme un lieu perpétuellement ouvert aux visiteurs : visitable en 
tout instant et de partout. D'autre part, il est également ouvert par sa forme évolutive et participative, proposant désormais une interface vivante entre l'artiste et son public. L'œuvre de l'artiste, habituellement visible par fragments et au cours des expositions successives (dispersées dans le temps et dans l'espace des différentes galeries et centres d'art), est ici saisissable comme un « tout » disposé en un même lieu.

Ces caractéristiques de l'Internet font qu'il est aujourd'hui possible d'engager l'expérimentation artistique en dehors des institutions et dispositifs officiels d'évaluation et de valorisation de l'art contemporain. Comme nous l'avons montré, le site s'appuie sur une culture partagée du réseau et de l'Internet créatif pour combiner ces différents éléments et les mettre au service d'une création collective. Exemplaire des mutations du travail artistique à l'œuvre dans le Net art, Mouchette promeut une redéfinition des conventions qui organisent la circulation et la réception des œuvres. Notre analyse s'est nourrie de ces distinctions, en pointant les ressorts de l'invitation préalable induite par l'œuvre, la conduite des expériences engagées et les conditions de leur partage. En se jouant des frontières entre les mondes, par l'absence de revendication et de signature artistique des productions pour Internet, Mouchette opère ainsi un contournement du circuit de l'art contemporain. Mais la contrepartie n'en est pas moins marquante : si depuis la création de son site Web en 1996 Mouchette a pu participer à de nombreuses manifestations artistiques en ligne, ses relations avec les mondes de l'art hors de l'Internet sont plus complexes. Les obstacles tiennent autant à la forme spécifique de cette œuvre Net art - intangible et virtuelle, difficile à extraire de l'ordinateur et $\mathrm{du}$ réseau Internet pour la transporter dans l'espace physique des galeries et des musées - qu'à l'inertie institutionnelle face à un projet artistique qui déjoue les normes traditionnelles d'homologation des œuvres et de définition du statut d'artiste. Mouchette est-elle l'œuvre ? Ou est-ce l'artiste qui crée les œuvres au sein de l'œuvre générique qui porte son nom ? Le projet est 
une mise en abîme qui empêche d'identifier clairement les frontières de l'œuvre et l'auteur auquel l'attribuer.

« On ne peut pas dire de Mouchette si elle est l'œuvre ou si elle est l'auteur. Cela brouille les pistes. Le fait que l'auteur soit inconnu sert à créer l'illusion, à créer un rapport direct. De la même manière que le montreur de marionnette fabrique une petite maison où la marionnette a l'air de vivre de sa vie propre. Plus on conserve l'illusion et plus on a de plaisir. C'est là le but de l'anonymat de l'artiste. Mais "Mouchette n'est pas une personne", c'est une œuvre, et je ne suis pas Mouchette. Quand je réponds à l'e-mail ça fait partie de l'œuvre. Quand *bisous* est intégré dans la signature, ce n'est pas moi qui l'écrit, c'est généré automatiquement. » (Entretien avec Mouchette, 2003.)

Mouchette ne se manifeste en effet au public, et par extension aux mondes de l'art, qu'au travers d'un avatar virtuel : le site ne faisant nullement mention de l'identité de son créateur. Cette inscription dans une tradition de l'anonymat et du doute sur l'identité se double ici d'un art de la parodie, d'une œuvre antérieure (le « Mouchette » de Robert Bresson) et d'une pratique plus populaire, l'écriture de journaux intimes, aujourd'hui supplantée par les nouvelles «pages perso » sur Internet. Dans ce contexte, l'anonymat de Mouchette est la condition de félicité de l'œuvre : l'identité artistique, jalousement gardée, se confond avec l'identité du personnage. Comme le dit la critique d'art Annick Bureaud dans un numéro de la revue Art Press (2000), « Mouchette se singularise par deux aspects : la fiction est énoncée ; seul le personnage est public, nous ignorons qui est l'individu qui a "inventé" Mouchette. Ainsi, nous pouvons communiquer par e-mail avec Mouchette, mais on ne la rencontre pas ». Si le flou identitaire et l'anonymat de l'auteur, comme du personnage, restent des figures artistiques connues, aussi nombreuses en littérature que dans la pratique des arts médiatiques (de l'art vidéo, notamment), elles se trouvent renouvelées par le contexte de l'Internet. Dans les rares articles et interviews que donne Mouchette, le plus souvent en ligne, la technologie du courriel<mmouchette@mouchette.org> 
permet ainsi d'entretenir le mystère et de ne rien dévoiler de l'identité d'artiste, tout en entretenant une relation soutenue avec son public. Mais ce qui apparaissait jusque-là comme une stratégie consciente et organisée de mise en œuvre d'art pour le réseau Internet constitue également le frein principal de sa diffusion et de son appropriation hors du réseau par les circuits habituels de l'art contemporain.

«Comme j'étais déjà artiste fonctionnant dans les réseaux de l'art contemporain (galeries, musées, commandes d'art public), j'étais assez détachée, critique mais plutôt amusée, quant à l'incapacité des institutions en place à prendre en compte cette forme artistique spécifique. Je fonctionnais sur deux régimes : artiste "normal" dans la journée et artiste du Net le soir derrière mon écran. Alors la situation me faisait rire et je n'en souffrais pas beaucoup. Par ailleurs, de manière pratique, j'ai pu bénéficier des bourses des Pays-Bas où je vis. J'ai donc pu pratiquer l'art sur le Net comme une recherche artistique sans avoir à fournir un produit tangible et commercialisable. » (Entretien par courriel, Mouchette, 2006.)

N'ayant rien à vendre, nul objet ni spectacle, à l'instar de nombreux projets Net art, son existence résulte - et dépend fortement - d'une économie du soutien public. Pour Mouchette, la reconnaissance dans le monde de l'art passe par l'attribution d'aides directes à la production (le curriculum vitae de Mouchette en fait amplement mention) et par des formes indirectes de rémunération du travail artistique (bourse, subvention, résidences de création) ou de sa transmission à des fins pédagogiques (comme conférencier et professeur invité dans des écoles d'arts ou des départements universitaires en arts plastiques et arts appliqués). L'auteur de Mouchette dont nous avons ici, suite à ses recommandations, choisi de respecter l'anonymat se trouve ainsi confronté au dilemme de la (non-)définition de son activité comme « artistique » et peut connaître par conséquent quelques difficultés, dans le cas de Mouchette, à vivre, à façonner et à affirmer un statut d'artiste traditionnel : du fait de la complexité de la proposition identitaire qui caractérise Mouchette, du fait également de son déploiement 
exclusif sur Internet qui reste encore, à bien des égards, un environnement expérimental dans lequel peu d'œuvres reçoivent le label institutionnel de la qualité artistique. Pourtant, si Mouchette se rit de la « labellisation » comme œuvre d'art, elle a néanmoins besoin de moyens de diffusion, de production et de conservation que les institutions ne sont pas à même de lui offrir. Que cela soit souhaité ou regretté, le fait est patent, si l'intérêt d'une politique artistique institutionnelle désireuse d'accompagner et de valoriser un art « en train de se faire » vient à se développer, les spécificités de l'art conçu pour et avec le réseau Internet - un art étranger au régime de l'objet original ou dérivé - s'accommodent mal des conventions de mise en exposition et de conservation sur lesquelles s'est fondé l'art moderne et contemporain.

\section{- Lexique du Web 2.0}

Blog : contraction de Web et Log (les journaux de bord de la marine et de l'aviation américaines), le blog est un journal en ligne permettant l'échange de points de vue.

Blogosphère : désigne l'ensemble de la communauté qui anime des blogs. Elle est généralement tissée d'une multitude de liens croisés, ou blogroll.

Crowdsourcing : en français, traitement par la foule. Consiste, pour les éditeurs de sites, à utiliser les internautes pour créer des contenus, répondre aux questions d'autres visiteurs, voire participer à la conception du site, d'un produit ou d'un service.

Flux (ou fils) rss ou Atom : formats populaires de syndication de contenus Web. Après s'être abonné à un flux sur un site, l'internaute reçoit les mises à jour de ce site automatiquement sur son ordinateur.

Folksonomy : contraction des mots folks (potes) et taxonomy (en français, la science du classement). Méthode de classification collaborative de contenus Web, via des tags, réalisée par une communauté d'internautes.

Podcasting : moyen de diffusion de fichiers audiovisuels (podcasts) sur le Net. Le podcast, une fois publié en ligne, est téléchargé automatiquement et transféré, via un fil rss, vers un ordinateur ou un baladeur numérique. 
Social bookmarking : partage de favoris ou de signets, en français, par leur enregistrement puis leur repérage par des tags sur un site Web public.

Syndication : consiste à intégrer sur un site, de manière automatique, tout ou partie du contenu d'un autre site. Ce procédé repose sur l'usage de fils rss ou Atom, deux formats de flux d'information auxquels les sites et les particuliers s'abonnent.

Tags : en français, étiquettes ou mots-clés. Proposés par le producteur de contenu, ils permettent de repérer et d'identifier des contenus dans la base de données.

Wiki : outil de gestion collaboratif de site Web qui permet aux internautes de rédiger et de modifier librement des contenus.

\section{NOTES}

[1] Cf. Mouchette http://www.mouchette.org.

[2] Le film Mouchette de Robert Bresson (1967) est lui-même une adaptation cinématographique du roman de Georges Bernanos.

[3] À l'instar de Mouchette, de nombreux projets Net art ont adopté la forme d'un récit évolutif proche du journal personnel et intime, mais désormais éditorialisé, donné à voir et à vivre en quasi-direct sur le Web. Ils déclinent en ligne les potentialités d'une archive qu'il est possible d'afficher et d'entretenir sur le long terme, en ayant ou non recours à la participation des visiteurs : cf. Jenni, JenniCam, http://www.jennicam.org, voir également Voog, Anna Clara, Anacam http://www.anacam.com et Merritt, Natacha, Digital diaries http://www.digital-diaries.com, Agnès de Cayeux, In my room, http://www.agnesdecayeux.fr.

[4] En inscrivant sa pratique dans la lignée d'une esthétique de la communication, Mouchette définit comme espace plastique un cadre relationnel entre les individus et renouvelle le modèle de la performance d'artiste par l'accomplissement d'un speech act (acte de langage), fruit de 
l'innovation (textuelle et culturelle) promue par l'écriture électronique en réseau. Elle donne ainsi forme à une création performative au double sens de l'« action » artistique et de l'acte de langage : sa propagation adopte les modes d'amplification propres à la rumeur qui juxtaposent une succession d'énoncés anonymes (se présentant comme de simples relais) et signés (pris en main par les différents relayeurs tout à la fois).

[5] Bien qu'il ne s'agisse que de la représentation virtuelle d'un chat, sur Internet, cliquer sur un mot est agir : le chat est mort !

[6] L'idée d'un " contrat de réception » entre l'artiste, l'œuvre et son public est empruntée à Jean-Claude Passeron et Emmanuel Pedler (1991) ou à Pedler (1994), et dans le domaine de l'esthétique à Nelson Goodman (1996) ou à Gérard Genette (1996).

[7] La notion de «prise » a été proposée par Christian Bessy et Francis Chateauraynaud (1995) pour rendre compte, sur un mode actif, de la saisie perceptuelle et de l'estimation des objets.

[8] La mise en demeure pour « diffusion illégale », datée du 23 juillet 2002, reproche à l'auteur du site Mouchette de profiter de la notoriété du film et du personnage inventé par Robert Bresson, diffusé sans autorisation et dénaturé dans un Quiz perçu comme « vraiment très éloigné du film qui traite d'un sujet grave ». Cf. http://mouchette.org/film/sacd.html.

[9] Extrait du message « posté » en août 2002 sur la liste Nettime (nnettimefr@ samizdat. net) : «The activities in support of mouchette.org will be announced at the following websites, where the urls of the several copies will also be constantly updated :

http://artlibre.org/events.php/miroirs/mouchette.html, http://www.constantvzw.com/copy.cult/mouchette. The information in English will be available here : http://drivedrive.com/mouchette/censored. html. You can download the forbidden work here and install it on your own site : http://www.constantvzw.com/copy.cult/ mirror/ ». 


\section{BIBLIOGRAPHIE}

Akrich Madeleine, 1990, « De la sociologie des techniques à une sociologie des usages », Techniques et Culture, $16: 83-110$.

Akrich Madeleine, 1993, «Les objets techniques et leurs utilisateurs. De la conception à l'action », in Bernard Conein, Nicolas Dodier et Laurent Thévenot (dir.), Les objets dans l'action, Paris, ehess, coll. « Raisons pratiques », $4: 35-58$.

Austin John, 1970, Quand dire c'est faire, Paris, Le Seuil.

Barthes Roland, 1975, « En sortant du cinéma », in Le bruissement de la langue. Essais critiques IV, Paris, Le Seuil : 407-412.

Becker Howard, 1988, Les mondes de l'art, Paris, Flammarion.

Bessy Christian, Francis Chateauraynaud, 1995, Experts et faussaires. Pour une sociologie de la perception, Paris, Métailié.

Bureaud Annick, 2000, « Mouchette », Art Press, 257.

Buscatto Marie, 2006, «Ethnographies du travail artistique : apports et usages épistémologiques », Sociologie de l'art, Opus 9-10: 89-105.

Certeau Michel de, 1990, L'invention du quotidien, tome 1: Arts de faire, Paris, Gallimard, coll. « Folio/Essais ».

Cochoy Frank, 2004 (dir.), La captation des publics. C'est pour mieux te séduire, mon client..., Toulouse, Presses universitaires du Mirail.

Conein Bernard, Nicolas Dodier, Laurent Thévenot (dir.), 1993, Les objets dans l'action, Paris, ehess, coll. « Raisons pratiques », 4.

Collins Harry, Michael Kush, 1998, The Shape of Actions. What Human and Machines Can Do, Cambridge, mit Press.

Davallon Jean, 1999, L'exposition à l'œuvre. Stratégies de communication et médiation symbolique, Paris, L'Harmattan.

Dodier Nicolas, 1993, "Les arènes des habiletés techniques », in Bernard Conein, Nicolas Dodier et Laurent Thévenot (dir.), Les objets dans l'action, Paris, ehess, coll. « Raisons pratiques », $4: 115-139$. 
Duguet Anne-Marie, 1988, « Dispositifs », Communications, Paris, Le Seuil : 221-242.

Duguet Anne-Marie, 2002, Déjouer l'image, Nîmes, Jacqueline Chambon.

Eco Umberto, 1965, L'œuvre ouverte, Paris, Le Seuil.

Fourmentraux Jean-Paul, 2003, L'œuvre en actes - Sociologie du Net art, thèse de doctorat de $3 \mathrm{e}$ cycle, Université de Toulouse II.

Fourmentraux Jean-Paul, 2005, Art et Internet. Les nouvelles figures de la création, Paris, cnrs Éditions.

Genette Gérard, 1996, L'œuvre de l'art. La relation esthétique, Paris, Le Seuil.

Goffman Erving, 1991, Les cadres de l'expérience, Paris, Éditions de Minuit.

Goodman Nelson, 1996, L'art en théorie et en action, Paris, Éditions de l'Éclat.

Heinich Nathalie, 1991, « Pour introduire à la cadre analyse », Critique, 535.

Hennion Antoine, 1993, La passion musicale - Une sociologie de la médiation, Paris, Métailié.

Hennion Antoine, Sophie Mainsonneuve, Émilie Gomart, 2000, Figures de l'amateur. Formes, objets, pratiques de l'amour de la musique aujourd'hui, Paris, La Documentation française.

Hughes Everett, 1996, Le regard sociologique, Paris, Éditions de l'ehess.

Kopytoff Igor, 1986, « The Cultural Biography of Things : Commodization as Process », in A. Appadurai (ed.), The Social Life of Things. Commodities in Cultural Perpective, Cambridge University Press.

Latour Bruno, 1994, "Une sociologie sans objet ? Remarques sur l'interobjectivité », Sociologie du travail, $4: 587-608$.

Latour Bruno, 1999, "From the concept of network to the concept of attachment », res, 36 .

Livingstone Sonia, Peter Lunt, 1993, «Un public actif, un téléspectateur critique », Hermès, 11-12: 145-158. 
Menger Pierre-Michel, 2002, Portrait de l'artiste en travailleur. Métamorphoses du capitalisme, Paris, Le Seuil, coll. « La République des idées $»$.

Norman Douglas, 1993, "Les artefacts cognitifs », in Bernard Conein, Nicolas Dodier et Laurent Thévenot (dir.), Les objets dans l'action, Paris, ehess, coll. « Raisons pratiques », 4 : 15-34.

Passeron Jean-Claude, Emmanuel Pedler, 1991, Le temps donné aux tableaux, Marseille, cercom/imerec.

Pedler Emmanuel, 1994, «En quête de réception : le deuxième cercle », Réseaux, 68.

Souriau Étienne, 1956, «L'œuvre à faire », Bulletin de la Société française de philosophie, séance du 25 février 1956.

Strauss Anselm, 1992, La trame de la négociation, Paris, L'Harmattan. 